\title{
bHLH factors in muscle development: dead lines and commitments, what to leave in and what to leave out
}

\author{
Eric N. Olson and William H. Klein \\ Department of Biochemistry and Molecular Biology, The University of Texas M.D. Anderson Cancer Center, Houston, \\ Texas 77030 USA
}

In recent years, skeletal muscle has become an important model for understanding the mechanisms that regulate tissue-specific gene expression. The formation of skeletal muscle during embryogenesis involves commitment of mesodermal progenitors to the myogenic lineage and subsequent differentiation of skeletal myoblasts into terminally differentiated myotubes. Like many cell types, skeletal myoblasts do not express markers of terminal differentiation until they are forced to exit the cell cycle in response to environmental cues. Growth factor signals play a central role in regulating the program for muscle-specific transcription by maintaining myoblasts in a proliferative state that is nonpermissive for the expression of muscle-specific genes.

Analysis of the mechanisms that regulate muscle differentiation in tissue culture led to the discovery of the four skeletal muscle-specific regulatory factors, MyoD (Davis et al. 1987), myogenin (Edmondson and Olson 1989; Wright et al. 1989), Myf5 (Braun et al. 1989a), and MRF4 (Rhodes and Konieczny 1989; Miner and Wold 1990; Braun et al. 1990), each of which can activate skeletal muscle gene transcription when expressed ectopically in a variety of nonmuscle cell types. Although many mammalian cell type-specific transcription factors have been identified, the myogenic factors are unique in their abilities to orchestrate an entire program of tissuespecific transcription when introduced into diverse cell types. This activity led to the notion that these factors function as master regulators of muscle cell fate during development. However, recent studies in which these genes have been inactivated through homologous recombination in transgenic mice have resulted in surprising phenotypes (or lack thereof) and have necessitated a reevaluation of the potential roles of these factors in the control of determination and differentiation in the myogenic lineage. Here, we review the models that emerged from studies of the myogenic factors in tissue culture and reconsider the potential functions of these factors in the embryo in light of recent gene-targeting experiments. For more comprehensive reviews on the control of muscle gene expression, the reader is referred to several recent reviews (Olson 1990, 1993; Weintraub et al. 1991;
Buckingham 1992; Sassoon 1992; Emerson 1993; Wright 1992).

Myogenic bHLH proteins are regulators of myogenesis in tissue culture

The myogenic regulators MyoD, myogenin, Myf5, and MRF4 share $\sim 80 \%$ amino acid identity within a basic helix-loop-helix (bHLH) motif that mediates dimerization and DNA binding. Within their amino and carboxyl termini, which function as transcriptional activation domains, these factors show only limited sequence similarity. Myogenic bHLH regulatory genes have been identified in mammals, birds, frogs, sea urchins, insects, and nematodes (Olson 1990; Weintraub et al. 1991; Sassoon 1992; Emerson 1993). Whereas vertebrate genomes encode the four myogenic bHLH proteins, invertebrates appear to express only a single member of this myogenic regulatory gene family. Each of the invertebrate factors is as similar to the four vertebrate factors in the bHLH region as they are to each other. This remarkable conservation, combined with the finding that the myogenic factors from sea urchins (Venuti et al. 1991) and nematodes (Krause and Weintraub 1992) can activate myogenesis in mammalian cells, indicates that the mechanisms that regulate muscle gene expression are extremely ancient and have been conserved since skeletal muscles first emerged in primitive metazoa several hundred million years ago.

Myogenic bHLH proteins form heterodimers with ubiquitous bHLH proteins, known as E proteins, and activate the transcription of muscle-specific genes by binding to the E-box consensus sequence (CANNTG) in muscle gene promoters and enhancers. Because all of these factors bind the same DNA sequence and activate their own and one another's expression in transfected cells (see below), it has been difficult to ascertain from transfection experiments whether they possess identical or distinct activities.

Studies in tissue culture have led to a model in which the myogenic bHLH proteins activate muscle-specific transcription by binding to $\mathrm{E}$ boxes in muscle gene con- 
trol regions or by inducing the expression of intermediate myogenic regulators that can activate the expression of muscle genes through E-box-independent pathways. One such intermediate regulator is myocyte enhancer factor 2 (MEF2), which is encoded by a family of four genes with products that share homology within a MADS domain (Pollock and Treisman 1991; Yu et al. 1992). The observation that forced expression of myogenin or MyoD in nonmuscle cells can induce MEF2 mRNA and protein (Cserjesi and Olson 1991; Lassar et al. 1991; Martin et al. 1993) is consistent with the notion that MEF2 acts in a regulatory cascade downstream of myogenic bHLH proteins. However, the recent discovery that MEF2-binding sites are important for transcription of the mouse myogenin (Edmondson et al. 1992; Cheng et al. 1993; Yee and Rigby 1993) and the Xenopus myoD (Leibham et al. 1994) genes suggests that myogenic bHLH proteins and MEF2 participate in a regulatory circuit involving direct and indirect positive feedback loops. The possibility that certain MEF2 isoforms may also act upstream of the myogenic bHLH regulatory genes has also been suggested (Breitbart et al. 1993).

In addition to activating the program for muscle-specific transcription, myogenic bHLH proteins appear to integrate incoming growth factor signals and thereby determine whether a myoblast will divide or differentiate. Growth signals are received in the nucleus by these myogenic regulators through changes in their phosphorylation (Li et al. 1992b) or availability of their dimerization partners (Jen et al. 1992), and through direct interactions with c-Jun (Bengal et al. 1992) or possibly through competition with c-Jun for common coregulators ( $\mathrm{Li}$ et al. 1992a). When external concentrations of growth factors decline and these inhibitory inputs diminish, the myogenic regulators acquire the ability to arrest cell growth and activate the myogenic program. The recent discovery that myogenin and MyoD interact directly with the retinoblastoma gene product (Gu et al. 1993) defines a point in which these cell-specific trans-activators and the cell cycle machinery intersect and suggests mechanisms whereby the control of muscle-specific transcription and cell cycle progression can be coupled.

\section{Positive and negative autoregulatory interactions occur among the myogenic bHLH factors}

When myogenic bHLH proteins are expressed in transfected cells, they not only activate markers of terminal differentiation, but they also induce their own expression (Braun et al. 1989b; Thayer et al. 1989). The importance of these auto- and cross-regulatory interactions for activation of muscle gene expression remains to be established, but it is reasonable to speculate that they may be important for amplifying and maintaining the expression of these genes in committed muscle cells. The recent finding that the mouse myogenin gene promoter contains an $\mathrm{E}$ box that is important for expression of a myogenin-lac $Z$ transgene in embryonic limb buds also suggests the existence of direct regulatory interactions among myogenic bHLH proteins in the embryo (Cheng et al. 1993; Yee and Rigby 1993).

Whereas Myf5 can activate MyoD expression in transfected fibroblasts, there appears to be a reciprocal relationship between the expression of MyoD and Myf5 in many muscle cell types. The possibility that MyoD and Myf5 might inhibit one another's expression is suggested by the observation that either MyoD or Myf5, but not both, seem to be expressed in established muscle cell lines. In addition, whereas the myoblast mutant cell line NFB expresses Myf5 at high levels, it does not express MyoD, and transfection of NFB cells with a MyoD expression vector results in a decline in Myf5 expression (Peterson et al. 1990). Whether this antagonism is mediated through direct interactions of $\mathrm{MyoD}$ and Myf5 with the corresponding gene control regions remains to be determined.

\section{MyoD and Myf5 are expressed in committed myoblasts, whereas myogenin and MRF4 are expressed only in postmitotic muscle cells}

As a general rule, either MyoD or Myf5 is expressed in proliferating myoblasts, whereas myogenin and MRF4 are not expressed until myoblasts exit the cell cycle in response to mitogen depletion. Myogenin expression has been detected in all muscle cell types at the time that they differentiate. In later stage muscle fibers, MRF4 becomes up-regulated and myogenin expression declines. Thus, MyoD and Myf5 may play similar roles in proliferating myoblasts, whereas myogenin and MRF4 may be required for activation and maintenance of muscle gene expression. Because these four proteins bind the same DNA sequence with comparable affinities, it is unclear how they might selectively activate different gene sets in myoblasts and myotubes. One possibility is that their unique amino and carboxyl termini may be important for specific protein-protein interactions with other transcription factors that bind different muscle gene control regions. Domain-swapping experiments suggest that these domains may allow these proteins to discriminate between different target genes (Chakraborty and Olson 1991; Mak et al. 1992). Identifying the specific gene targets for each of the myogenic bHLH regulators is an important problem for the future.

\section{The myogenic regulators show unique expression patterns in vivo}

Skeletal muscle in vertebrates is derived from the somites, which form in a rostral-to-caudal fashion by segmentation of the paraxial mesoderm along the neural tube (for review, see Buckingham 1992). As they mature, the somites first become compartmentalized into the sclerotome and dermamyotome, which give rise to cartilage and bone, and appendicular muscle, respectively. Subsequently, the dermamyotome separates into the myotome and dermatome, which give rise to axial skeletal muscle and the dermis. Myf5 is the first of the myogenic regulatory genes to be expressed during mouse em- 
bryogenesis, with transcripts appearing in the somite at day 8.0 postcoitum (p.c.), immediately prior to somite compartmentalization. Myogenin transcripts appear in the myotome by day 8.5 p.c., and MRF4 and MyoD are expressed in the myotome beginning at day 9.0 and 10.5 p.c., respectively. In the limb buds, Myf5 transcripts appear at day 11.0 p.c., with myogenin and MyoD mRNAs expressed about half a day later. MRF4 mRNA does not accumulate in muscle fibers in the limbs until birth, when it is up-regulated and becomes the predominant member of the family to be expressed. Whereas Myf5 is expressed before MyoD in mouse embryos, the order of expression is reversed in birds (Pownall and Emerson 1991). These interchangeable expression patterns raise the possibility that these proteins may be functionally interchangeable as well.

\section{MyoD is not required for myogenesis in the mouse embryo}

A central issue is whether the activities of the mammalian myogenic bHLH regulators in tissue culture accurately reflect their functions in the embryo. To approach this question, several laboratories have targeted mutations to the individual myogenic regulatory genes through homologous recombination. Remarkably, MyoD-null mice are fully viable and show no obvious muscle abnormalities (Rudnicki et al. 1992). The only effect of $M y o D$ inactivation identified thus far is an approximate twofold increase in the level of Myf5 mRNA expression in skeletal muscle, consistent with the reciprocal regulation of these genes in tissue culture cells. The presence of normal levels of myogenin and MRF4 transcripts in these MyoD-null mice demonstrates that MyoD is not essential for activation of these genes.

It is possible that MyoD-null mice might exhibit defects in aspects of muscle function not examined thus far. For example, changes in MyoD expression have been implicated in responsiveness of muscle to innervation, thyroid hormone, and insulin-like growth factors (Eftimie et al. 1991; Florini et al. 1991; Hughes et al. 1993). Perhaps, these mice will be impaired in these responses or in other aspects of muscle maturation or regeneration. The recent finding that $\mathrm{MyoD}$ is preferentially expressed in fast-twitch muscle fibers (Hughes et al. 1993) also raises the possibility that subtle changes in fiber type distribution or expression of muscle protein isoforms may occur in the absence of MyoD. It is thus premature to conclude that other myogenic factors can substitute for all functions of MyoD.

\section{Targeting of Myf5 affects skeletal but not muscle development}

An understanding of the functions of the myogenic bHLH regulatory genes in the embryo was further clouded by the discovery that mice homozygous for a Myf5 mutation also develop normal skeletal muscle but die at birth as a result of the absence of the distal parts of the ribs, which results in an inability to breathe (Braun et al. 1992). The only apparent alteration within the skeletal muscle lineage in these mice is a 2-day delay in the initial expression of muscle markers in the myotomes. Considering that the somite represents the origin of skeletal muscle in vertebrates, it is remarkable that the complete complement of skeletal muscle fibers can develop following a 2-day delay in myotome formation.

Despite the fact that Myf5 is the first of the myogenic regulators to be expressed during embryogenesis, newborn mice lacking Myf5 express normal levels of transcripts for the other myogenic bHLH regulators. This suggests that myogenin, which is normally expressed immediately following Myf5 in the myotome and limb buds, does not require Myf5 for expression. However, until the temporal pattern of expression of $\mathrm{MyoD}$ or MRF4 is determined in the Myf5-null background, it remains possible that they become expressed earlier in the absence of Myf5 and thereby activate myogenin gene expression.

Why does Myf5 gene inactivation lead to a rib defect? One possibility is that formation of the distal portions of the ribs requires an inductive interaction between Myf5positive cells in the dermamyotome and the juxtaposed sclerotomal precursors from which the ribs are derived. Such interactions could conceivably involve the Myf5mediated release by myotomal cells of extrinsic factors required for differentiation of sclerotomal cells. In this regard, bone morphogenetic protein $2 \mathrm{~A}$, which can induce bone formation, has been localized to the somites of mouse embryos (Lyons et al. 1990), as have several members of the fibroblast growth factor family (Haub and Goldfarb 1991; Niswander and Martin 1992). It is also possible that myotomal cells secrete extracellular matrix molecules that are permissive for sclerotomal cell differentiation (Solursh et al. 1979). None of these possibilities, however, adequately explain why ribs are the only skeletal elements affected in the Myf5-mutant mice.

Although there has been no direct evidence for inductive interactions between different compartments of the somite, the importance of short-range inductive interactions for appropriate differentiation of somitic cell lineages is well established. The formation of vertebral bodies by sclerotomal cells, for example, is dependent on inductive interactions between the neural tube and the sclerotome (Hall 1977). Similarly, the neural tube appears to play an important role in the induction of myogenic cells within the somites (Rong et al. 1992).

An alternative explanation for the rib defect in Myf5null mice is that Myf5 may be transiently expressed in a common precursor cell of the sclerotome and myotome and thereby acts in a cell-autonomous manner to control rib development. At the time Myf5 expression is initiated, the somites have not yet become compartmentalized into the myotome, sclerotome, and dermatome. Thus, it is conceivable that Myf5 is transiently expressed in a sclerotomal progenitor and is then rapidly extinguished. However, as Myf5 expression has only been detected in the myotomal region of compartmentalized somites, this possibility is unlikely. The generation of 
chimeric mice containing different contributions of wild-type and Myf5-null cells should resolve whether Myf5 acts through a cell-autonomous mechanism to influence rib development.

Does the presence of normal skeletal muscle in MyoDand Myf5-null mice mean that the functions of these regulatory genes in skeletal muscle are redundant? The recent creation of mice lacking both MyoD and Myf5 suggests that the function of these genes are overlapping. MyoD -/-, Myf5 -/- mice produce no detectable muscle gene products (Rudnicki et al. 1993). That myoblast markers such as desmin also fail to be expressed in these mice suggests that MyoD and Myf5 act early in the myogenic lineage and determine myoblast identity. Whether inactivation of both genes prevents commitment of mesodermal progenitors to the myogenic lineage or prevents expression of muscle markers in committed myoblasts is difficult to determine.

\section{Myogenin is essential for normal muscle development in vivo but not in vitro}

In sharp contrast to $M y o D$ and Myf5, two recent reports have demonstrated that mice with targeted mutations in the myogenin gene display severe skeletal muscle deficiencies (Hasty et al. 1993; Nabeshima et al. 1993). myogenin-mutant animals survive fetal development but die perinatally, and muscle-forming regions throughout the bodies of mutant neonates and embryos have far fewer myofibers. Thus, the results of both studies provide dramatic evidence for the essential role of myogenin in muscle development. Although muscle differentiation is disrupted in myogenin-null mice, muscle tissues appear relatively normal at a gross level, indicating that myogenin is not required for positional information involved in muscle tissue morphogenesis.

In newborn mutant mice, regions where muscle would normally be present are populated by sparse myofibers and mononucleate cells that do not stain positively for myosin heavy-chain or filamentous actin, indicating that they are not differentiated muscle cells lacking the ability to fuse (Hasty et al. 1993). Residual myofibers with seemingly normal sarcomeric organization are routinely observed throughout the body of the mutant neonates. In addition, the neonates have significant skeletal deformities, although these may be secondary to the absence of skeletal muscle.

Nabeshima et al. (1993) described 13.5- and 14.5-day embryos and reported that the extent of muscle disorganization in mutant mice at these earlier stages differs in three regions. On the basis of their observations these investigators propose that migratory myogenic precursor cells are more dependent on myogenin than are cells that will form the myotome and, subsequently, axial muscles. Such differences were not observed in mutant neonates (Hasty et al. 1993).

The myogenin-mutant mice provide a way of discerning whether myogenin is strictly required for the expression of all muscle-specific genes or whether individual genes may be regulated by specific myogenic bHLH pro- teins. myogenin-mutant neonates do not express appreciable levels of transcripts for several muscle-specific structural genes, but they do express others (Hasty et al. 1993). These findings suggest that the expression of different muscle-specific genes can be uncoupled and that myogenin is essential for the regulation of a subset of genes in vivo. In addition, the mutant mice express reduced levels of MRF4 but normal levels of MyoD. Whether the diminished expression of MRF4 reflects a direct role for myogenin in regulating the MRF4 gene or whether cells producing MRF4 are absent or reduced remains to be determined. Most noteworthy is that myogenin is not strictly required for MyoD expression. In addition, because wild-type levels of $\mathrm{MyoD}$ transcripts are present in the myogenin-mutant neonates, MyoD is apparently not sufficient for normal muscle differentiation in the absence of myogenin. Cells within myogenic regions of mutant 14.5-day embryos contain MyoD protein at levels comparable with wild-type embryos, further implying that the absence of myogenin does not affect MyoD expression. The presence of MyoD in the cells of mutant animals implies that myogenin is not required for the commitment of cells to the myogenic lineage but, rather, is important for terminal differentiation.

The residual myofibers in the myogenin-mutant mice raise the possibility that certain muscle fiber types, for example, fast- or slow-twitch fibers, require myogenin for differentiation while others do not. Relevant to this is a recent report indicating that myogenin transcripts accumulate preferentially in slow-twitch muscles in the hindlimb of the adult rat while MyoD transcripts are enriched in fast-twitch muscles (Hughes et al. 1993). However, preliminary results using antibodies to different myosin heavy chain isoforms suggest that the residual myofibers present in the mutant embryos are not a subset of fast- or slow-twitch myofibers (J. Venuti, E. Olson, and W. Klein, unpubl.). Our view of the myogenin-mutant phenotype is that some aberrant myofibers missing essential muscle-specific gene products may form and subsequently degenerate. However, it is also apparent that the complete complement of myofibers fails to differentiate, and undifferentiated cells remain in their place. Finally, normal myofibers found in low numbers are always present despite the absence of myogenin.

Why does the myogenin mutant show such a profound muscle phenotype compared with the $M y o D$ and Myf5 mutants? One possibility is that the myogenin protein possesses functions not shared with MyoD or Myf5. If this is the case, this uniqueness would most likely be contained within the amino- or carboxy-terminal domains of myogenin, which are distinct from those of the other factors. Alternatively, myogenin may be expressed in muscle cells at a specific time and place during embryogenesis that is important for muscle formation. The expression pattern of myogenin in the embryo overlaps with but is distinct from those of the other myogenic regulators. The recent identification of DNA sequences upstream of the myogenin gene that direct myogenin expression during embryogenesis /Cheng et al. 1992, 
1993; Yee and Rigby 1993) should make it possible to test the latter possibility by placing other myogenic bHLH genes under control of the myogenin gene promoter and expressing them in place of myogenin within the myogenin-null mice.

The large numbers of unfused cells present where muscle fibers would normally be suggest that the absence of myogenin may block these cells from terminal differentiation. It would be interesting to know whether such cells are still capable of receiving external cues and exiting from the cell cycle or if they continue to actively proliferate. One approach to investigating the properties of the undifferentiated cells is to isolate and culture myoblasts from myogenin-mutant embryos. Surprisingly, myoblasts from mutant limbs behave identically to wild-type myoblasts. They proliferate normally under conditions of high serum, and, remarkably, they differentiate into myotubes when forced to exit the cell cycle (Nabeshima et al. 1993; D. Edmondson, W. Klein, and E. Olson, unpubl.). Differentiated myotubes from mutants show normal sarcomeric structure and undergo spontaneous contraction, implying that they express normal levels of contractile proteins and other terminal musclespecific gene products not seen in the mutant animals. We are thus left with the interesting conclusion that the mutant myoblasts are competent for terminal differentiation in the absence of myogenin, but they are somehow suppressed from doing so in vivo.

The ability of myogenin-null myoblasts to differentiate in vitro, but not in vivo, suggests that the environment of the embryo is not permissive for the other myogenic regulators to activate the myogenic program. Perhaps the block to myogenesis in vivo is mediated by influences such as exogenous growth factors, the extracellular matrix, or cell-cell interactions, which are lost in culture. It will also be interesting to determine whether one or more of the three remaining myogenic bHLH proteins become upregulated when myogeninnull myoblasts are placed in culture and thereby compensate for the absence of myogenin, at least in vitro.

\section{Genes encoding myogenic bHLH factors in invertebrate organisms can be deleted without preventing muscle gene expression}

Because the myogenic bHLH factors are conserved in species spanning vast phylogenetic distances, organisms outside of vertebrates offer a particularly useful comparative approach for studying the functions of these factors. In Caenorhabditis elegans, the cell lineages leading to the 85 striated body wall muscle cells can be traced precisely back to four of the six founder cells of the early embryo. Unlike vertebrate skeletal muscle formation, where extrinsic influences are essential, at least some of the $C$. elegans body wall muscle cells form in a cellautonomous fashion, suggesting the segregation of an early determinant during embryogenesis. Although the initial commitment step appears to be quite different, $C$. elegans nonetheless contains a myogenic bHLH factor, CeMyoD, which is stably expressed in all body wall muscle cells shortly after each cell arises (Krause and Weintraub 1992). From the expression patterns in vivo and myogenic activity in mammalian fibroblasts, it is likely that CeMyoD is an important regulator of muscle gene expression in body wall muscle cells. Surprisingly, animals homozygous for small deficiencies that remove the $C e M y o D$ gene make the correct number of body wall muscle cells, although sarcomeric organization is somewhat disorganized compared with that of wild-type muscle (Chen et al. 1992). Although the deficient strains are recessive embryonic lethals, it is probable that lethality arises from other genes deleted from this region. A minimum conclusion from these results is that zygotic expression of CeMyoD is not required for development of body wall muscle. Because it is unlikely that a related bHLH gene exists in C. elegans, these results are difficult to assess in light of current thinking about the role of the myogenic bHLH regulators. One possibility is that the homozygous mutant embryos have a low level of maternal CeMyoD (or CeMyoD mRNA) that is sufficient to initiate the myogenic program. A second explanation is that the genetic pathways leading to muscle differentiation "downstream" of CeMyoD can be activated by other regulatory genes unrelated to CeMyoD.

In Drosophila, muscle cells are derived from mesoderm cells that invaginate along the ventral midline. A single bHLH factor, encoded by a gene termed nautilus or Dmyd, has homology to the mammalian myogenic factors and is expressed transiently in a subset of myogenic precursor cells that may represent the founder myoblasts of each segment (Michelson et al. 1990; Paterson et al. 1991). Recent experiments indicate that Drosophila embryos containing homozygous deficiencies that delete the nautilus gene land perhaps other surrounding genes) show disorganized muscle but, nevertheless, express the muscle-specific structural genes actin and myosin (S. Abmayr, R. Drysdale, A. Michelson, and M. Bate, pers. comm.). Thus, nautilus, like $C e M y o D$, is not strictly required for muscle gene expression.

A potential explanation for muscle gene expression in the absence of nautilus may lie in MEF2, which is expressed in muscle cell precursors prior to nautilus during Drosophila embryogenesis (B. Lilly, S. Galewsky, A.B. Firulli, and R. Schulz, unpubl.; H. Nguyen and B. NadalGinard, pers. comm.). Because MEF2 can activate muscle gene expression in the absence of myogenic bHLH proteins (Gossett et al. 1989; Yee and Rigby 1993), it may partially activate the myogenic program in the absence of nautilus. Perhaps a similar mechanism accounts for muscle gene expression in C. elegans mutants lacking CeMyoD.

\section{What is the function of the myogenic bHLH proteins in development?}

Based on gain-of-function assays in tissue culture, it is clear that the myogenic bHLH proteins can activate the complete myogenic program. However, whether these proteins function at the determination step, to mediate the initial commitment of mesodermal stem cells to the 


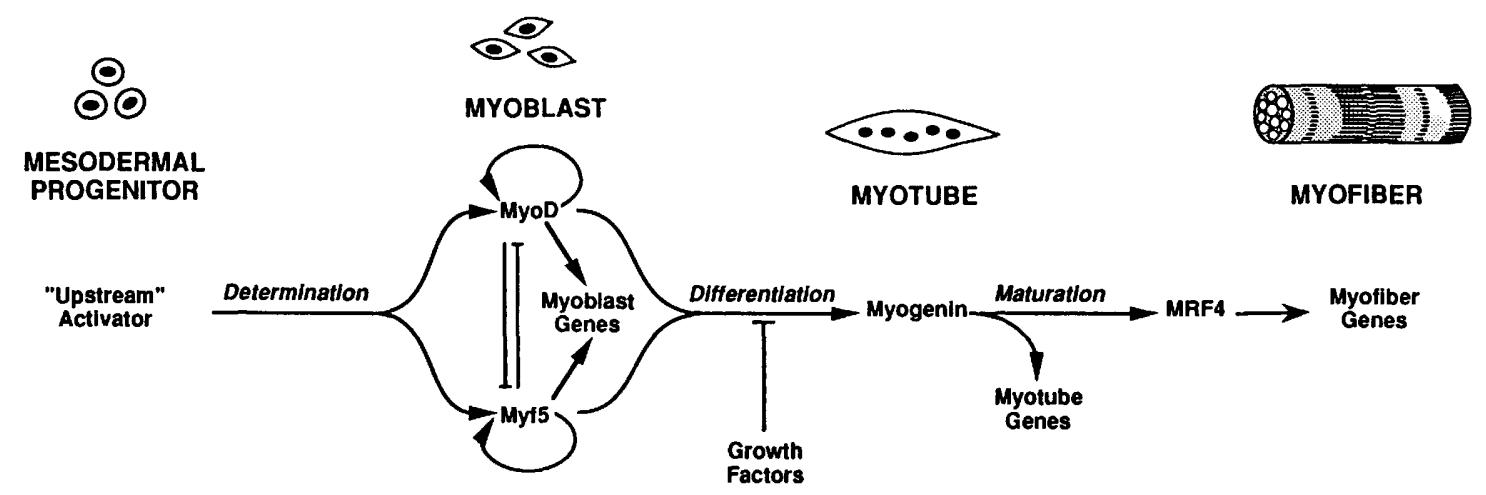

Figure 1. Hypothetical regulatory pathway for muscle determination and differentiation. According to this model, either Myob or Myf 5 become expressed as a consequence of myogenic lineage determination. MyoD and Myf5 autoactivate their expression, but they may negatively regulate one another. Myoblast-specific genes would be regulated in proliferating myoblasts by MyoD or Myf5. Upon depletion of exogenous growth factors, MyoD or Myf5 would activate (directly or indirectly) expression of myogenin, which activates myotube-specific genes. During myofiber maturation, myogenin expression declines and MRF4 is up-regulated to control myofiberspecific genes. The model does not take into account potential changes in regulation of the myogenic factors that may occur when one is removed from the regulatory circuitry by homologous recombination.

myogenic lineage, or act as muscle-specific transcription factors that activate terminal differentiation when extracellular influences are permissive for cell cycle withdrawal, has been debated. From the gene-targeting experiments reported thus far, we can begin to place these genes within a genetic pathway leading to myogenesis. Because mutations in $M y f 5$ and $M y o D$ have no observable effect on muscle formation, whereas mutations in myogenin have strong effects, and because Myf5 and MyoD are often expressed prior to myogenin in muscle cells in vitro, it is likely that myogenin lies downstream of Myf5 and MyoD. This is not meant to imply that the pathway to muscle differentiation is linear but only that certain early events of muscle differentiation require either Myf5 or MyoD while certain later events require myogenin. As discussed above and depicted in Figure 1, Myf5 and MyoD may perform similar functions in committed myoblasts. The fact that MyoD is expressed later than Myf5 in development may simply reflect temporal differences in individual populations of myoblasts. Some myoblasts may initiate their differentiation program early and utilize Myf5, whereas later populations use MyoD. The absence of muscle markers in double homozygous $M y f 5 / M y o D$ mutant mice is also consistent with a common early function of Myf5 and MyoD and indicates that myogenin is unable to perform the "upstream" functions normally performed by these factors. We think it unlikely that either Myf5 or MyoD is necessary for the initial commitment of the pluripotent mesoderm cell to a myogenic fate, as myogenic lineage commitment appears to be a stable decision, whereas the expression of these myogenic regulators is subject to regulation by activated oncogenes and 5-bromo-2'-deoxyuridine, which inhibit myogenesis (Lassar et al. 1989; Tapscott et al. 1989). Moreover, there is substantial evidence for the existence of committed myogenic cells in limb buds of vertebrate embryos that do not express any of the myogenic bHLH regulators (Sassoon et al. 1989).
Rather, MyoD and Myf5 may be expressed as a consequence of myogenic lineage commitment and may be involved in maintaining myoblast identity by regulating myoblast-specific genes. The recent demonstration that MyoD activates myogenin gene expression but not markers of terminal differentiation is consistent with this view (Hollenberg et al. 1993). Accordingly, myogenin could act as a necessary regulator downstream of these early events and could be directly activated by Myf 5 or MyoD as well as by alternative mechanisms yet to be identified. It is possible that MRF4 plays the same role as myogenin after birth. It is also possible that MRF4 is downstream of myogenin in the muscle differentiation program. Either possibility would predict that an MRF4mutant mouse would show skeletal muscle deficiencies.

Because the myogenic bHLH proteins function within a network of regulators, it is not unreasonable to expect that non-bHLH proteins, such as MEF2, which function in this network, may also be altered when the regulatory balance among these genes is disrupted through gene targeting and may therefore constitute an alternate pathway leading to muscle gene activation. An important challenge for the future will be to identify and define these alternate pathways. Finally, as other cell-specific trans-activators become identified, it will be important to determine whether the regulatory strategies leading to myogenesis reflect common mechanisms for the control of cell fates in other lineages or whether the bHLH myogenic regulators represent a unique set of functions that have evolved solely for skeletal muscle.

\section{Acknowledgments}

We are most grateful to Paul Hasty, Judith Venuti, and Diane Edmondson for helpful comments on the manuscript and to $\mathrm{K}$. Tucker for editorial assistance. This work is supported by grants from the National Institutes of Health, the Muscular Dystrophy Association, and the Robert A. Welch Foundation. 


\section{References}

Bengal, E., L. Ransone, R. Scharfmann, V.J. Dwarki, S.J. Tapscott, H. Weintraub, I.M. Verma. 1992. Functional antagonism between c-Jun and MyoD proteins: A direct physical association. Cell 68: 507-519.

Braun T., D.G. Buschhausen, E. Bober, E. Tannich, and H.H. Arnold. 1989a. A novel human muscle factor related to but distinct from MyoDl induces myogenic conversionin 10T1/2 fibroblasts. EMBO I. 8: 701-709.

Braun, T., E. Bober, D.G. Buschhausen, S. Kohtz, K.H. Grzeschik, and H.H. Arnold. 1989b. Differential expression of myogenic determination genes in muscle cells: Possible autoactivation by the Myf gene products. EMBO I. 8: 36173625.

Braun, T., E. Bober, B. Winter, N. Rosenthal, and H.H. Arnold. 1990. Myf-6, a new member of the human gene family of myogenic determination factors: evidence for a gene cluster on chromosome 12. EMBO I. 9: 821-831.

Braun, T., M.A. Rudnicki, H.-H. Arnold, and R. Jaenisch. 1992. Targeted inactivation of the muscle regulatory gene $M y f-5$ results in abnormal rib development and perinatal death. Cell 71: 369-382.

Breitbart, R., C. Liang, L. Smoot, D. Laheru, V. Mahdavi, and B. Nadal-Ginard. 1993. A fourth human MEF-2 transcription factor, hMEF2D, is an early marker of the myogenic lineage. Development 118: 1095-1106.

Buckingham, M. 1992. Making muscle in mammals. Trends Genet. 8: 144-148.

Chakraborty, T. and E.N. Olson. 1991. Domains outside of the DNA-binding domain impart target gene specificity to myogenin and MRF4. Mol. Cell. Biol. 11: 6103-6108.

Chen, L., M. Krause, B. Draper, H. Weintraub, and A. Fire. 1992. Body-wall muscle formation in Caenorhabditis elegans embryos that lack the MyoD homolog hlh-1. Science 256: 240243.

Cheng, T.-C., T.A. Hanley, J. Mudd, J.P. Merlie, and E.N. Olson. 1992. Mapping of myogenin transcription during embryogenesis using transgenes linked to the myogenin control region. J. Cell Biol. 119: 1649-1656.

Cheng, T.-C., M. Wallace, J.P. Merlie, and E.N. Olson. 1993. Separable regulatory elements govern myogenin transcription in embryonic somites and limb buds. Science 261: 215218.

Cserjesi, P. and E.N. Olson. 1991. Myogenin induces musclespecific enhancer binding factor MEF-2 independently of other muscle-specific gene products. Mol. Cell. Biol. 11: 4854-4862.

Davis, R.L., H. Weintraub, and A.B. Lassar. 1987. Expression of a single transfected cDNA converts fibroblasts to myoblasts. Cell 51: 987-1000.

Edmondson, D.G. and E.N. Olson. 1989. A gene with homology to the myc similarity region of MyoD1 is expressed during myogenesis and is sufficient to activate the muscle differentiation program. Genes \& Dev. 3: 628-640.

Edmondson, D.G., T.-C. Cheng, P. Cserjesi, T. Chakraborty, and E.N. Olson. 1992. Analysis of the myogenin promoter reveals an indirect pathway for positive autoregulation mediated by the muscle-specific enhancer factor MEF-2. Mol. Cell. Biol. 12: 3665-3677.

Eftimie, R., H.R. Brenner, and A. Buonanno. 1991. Myogenin and MyoD join a family of skeletal muscle genes regulated by electrical activity. Proc. Natl. Acad. Sci. 88: 1349-1353.

Emerson, C.P. 1993. Skeletal myogensis: Genetics and embryology to the fore. Curr. Opin. Genet. \& Dev. 3: 265-274.

Florini, J.R., D.Z. Ewton, and S.L. Roof. 1991. Insulin-like growth factor-I stimulates terminal myogenic differentiation by induction of myogenin gene expression. Mol. Endocrinol. 5: 718-724.

Gossett, L.A., D.J. Kelvin, E.A. Sternberg, and E.N. Olson. 1989 A new myocyte-specific enhancer-binding factor that recognizes a conserved element associated with multiple musclespecific genes. Mol. Cell. Biol. 9: 5022-5033.

Gu, W., J.W. Schneider, G. Condorelli, S. Kaushal, V. Mahdavi, and B. Nadal-Ginard. 1993. Interaction of myogenic factors and the retinoblastinoma protein mediates muscle cell commitment and differentiation. Cell 72: 309-324.

Hall, B.K. 1977. Chondrogenesis of the somitic mesoderm. Adv. Anat. Embryol. Cell Biol. 53: 1-50.

Hasty, P., A. Bradley, J.H. Morris, D.G. Edmondson, J. Venuti, E.N. Olson, and W.H. Klein. 1993. Muscle deficiency and neonatal death in mice with a targeted mutation in the myogenin gene. Nature 364: 501-506.

Haub, O. and M. Goldfarb. 1991. Expression of the fibroblast growth factor 5 gene in the mouse embryo. Development 112: $397-406$.

Hollenberg, S.M., P.F. Cheng, and H. Weintraub. 1993. Use of a conditional MyoD transcription factor in studies of $\mathrm{MyoD}$ trans-activation and muscle determination. Proc. Natl. Acad. Sci. 90: 8028-8032.

Hughes, S.M., J.M. Taylor, S.J. Tapscott, C.M. Gurley, W.J. Carter, and C.A. Peterson. 1993. Selective accumulation of MyoD and myogenin mRNAs in fast and slow adult skeletal muscle is controlled by innervation and hormones. Development 118: 1137-1147.

Jen, Y., H. Weintraub, and R. Benezra. 1992. Overexpression of Id protein inhibits the muscle differentiation program: In vivo association of Id with E2A proteins. Genes. \& Dev. 6: 1466-1479.

Krause, M. and H. Weintraub. 1992. CeMyoD expression and myogenesis in C. elegans. Semin. Dev. Biol. 3: 277-285.

Lassar, A.B., M.J. Thayer, R.W. Overell, and H. Weintraub. 1989. Transformation by activated RAS or FOS prevents myogenesis by inhibiting expression of MyoDl. Cell 58: 659-667.

Lassar, A.B., R.L. Davis, W.E. Wright, T. Kadesch, C. Murre, A. Voronova, D. Baltimore, and H. Weintraub. 1991. Functional activity of myogenic HLH proteins requires hetero-oligomerization with E12/E47-like proteins in vivo. Cell 66: 305315 .

Leibham, D., M. Wong, T.-C. Cheng, S. Schroeder, P.A. Weil, E.N. Olson, and M. Perry. 1994. Binding of TFIID and MEF2 to the TATA element activate transcription of the Xenopus MyoDa promoter. Mol. Cell. Biol. 14: (in press).

Li, L., J.-C. Chambard, M. Karin, and E.N. Olson. 1992a. Fos and Jun repress transcriptional activation by myogenin and MyoD: The amino terminus of Jun can mediate repression. Genes \& Dev. 6: 676-689.

Li, L., J. Zhou, G. James, R. Heller-Harrison, M. Czech, and E.N. Olson. 1992b. FGF inactivates myogenic helix-loop-helix proteins through phosphorylation of a conserved protein kinase C site in their DNA binding domains. Cell 71: 1181-1194.

Lyons, K.M., R.W. Pelton, and B.L.M. Hogan. 1990. Organogenesis and pattern formation in the mouse: RNA distribution patterns suggest a role for bone morphogenic protein-2A (BMP-2A). Development 109: 833-844.

Mak, K.-L., R.Q. To, Y. Kong, and S.F. Konieczny. 1992. The MRF4 activation domain is required to induce muscle-specific gene expression. Mol. Cell. Biol. 12: 4334-4346.

Martin, J.F., J.J. Schwarz, and E.N. Olson. 1993. Myocyte enhancer factor (MEF) 2C: A tissue-restricted member of the MEF-2 family of transcription factors. Proc. Natl. Acad. Sci. 90: 5282-5286. 
Michelson, A.M., S.M. Abmayr, M. Bate, A. Martinez Arias, and T. Maniatis. 1990. Expression of a $M y o D$ family member prefigures muscle pattern in Drosophila embryos. Genes. (4) Dev. 4: 2086-2097.

Miner, J.H. and B. Wold. 1990. Herculin, a fourth member of the $M y o D$ family of myogenic regulatory genes. Proc. Natl. Acad. Sci. 87: 1089-1093.

Nabeshima, Y., K. Hanaoka, M. Hayasaka, E. Esumi, S. Li, I. Nonaka, and Y. Nabeshima. 1993. Myogenin gene disruption results in perinatal lethality because of severe muscle defect. Nature 364: 532-535.

Niswander, L. and G.R. Martin. 1992. FGF-4 expression during gastrulation, myogenesis, limb and tooth development in the mouse. Development 114: 755-768.

Olson, E.N. 1990. The MyoD family, a paradigm for development? Genes \& Dev. 4: 1454-1461.

- 1993. Interplay between proliferation and differentiation within the myogenic lineage. Dev. Biol. 154: 261-272.

Paterson, B.M., U. Walldorf, J. Eldridge, A. Dübendorfer, M. Frasch, W.J. Gehring. 1991. The Drosophila homologue of vertebrate myogenic-determination genes encodes a transiently expressed nuclear protein marking primary myogenic cells. Proc. Natl. Acad. Sci. 88: 3782-3786.

Peterson, C.A., H. Gordon, Z.W. Hall, B.M. Paterson, and H.M. Blau. 1990. Negative control of the helix-loop-helix family of myogenic regulators in the NFB mutant. Cell 62: 493-502.

Pollock, R. and R. Treisman. 1991. Human SRF-related proteins: DNA-binding properties and potential regulatory targets. Genes. 4 Dev. 5: 2327-2341.

Pownall, M.E. and C.P. Emerson. 1991. Sequential activation of three myogenic regulatory genes during somite morphogenesis in quail embryos. Dev. Biol. 151: 67-79.

Rhodes, S.J. and S.F. Konieczny. 1989. Identification of MRF4: A new member of the muscle regulatory factor gene family. Genes \& Dev. 3: 2050-2061.

Rong, P.M., M.-A. Teillet, C. Ziller, and N.M. Le Douarin. 1992. The neural tube/notochord complex is necessary for vertebral but not limb and body wall striated muscle differentiation. Development 115: 657-672.

Rudnicki, M.A., T. Braun, S. Hinuma, and R. Jaenisch. 1992. Inactivation of $M y o D$ in mice leads to up-regulation of the myogenic HLH gene $M y f-5$ and results in apparently normal muscle development. Cell 71: 383-390.

Rudnicki, M.A., P.N.J. Schnegelsberg, R.H. Stead, T. Braun, H.H. Arnold, and R. Jaenisch. 1993. MyoD or Myf-5 is required for the formation of skeletal muscle. Cell (in press).

Sassoon, D.A. 1992. Myogenic regulatory factors: Dissection of their role and regulation during vertebrate embryogenesis. Dev. Biol. 156: 11-23.

Sassoon, D., G. Lyons, W.E. Wright, V. Lin, A.B. Lassar, H. Weintraub, and M. Buckingham. 1989. Expression of two myogenic regulatory factors myogenin and MyoDl during mouse embryogenesis. Nature 341: 303-307.

Solursh, M., M. Fisher, S. Meier, and C.T. Singley. 1979. The role of extracellular matrix in the formation of the sclerotome. J. Embryol. Exp. Morphol. 54: 75-98.

Tapscott, S.J., A.B. Lassar, R.L. Davis, and H. Weintraub. 1989. 5-Bromo-2'-deoxyuridine blocks myogenesis by extinguishing expression of MyoD1. Science 242: 405-411.

Thayer, M.J., S.J. Tapscott, R.L. Davis, W.E. Wright, A.B. Lassar, and $\mathrm{H}$. Weintraub. 1989. Positive autoregulation of the myogenic determination gene MyoDl. Cell 58: 241-248.

Venuti, J.M., L. Goldberg, T. Chakraborty, E.N. Olson, and W.H. Klein. 1991. A myogenic factor from sea urchin embryos capable of programming muscle differentiation in mammalian cells. Proc. Natl. Acad. Sci. 88: 6219-6223.
Weintraub, H., R. Davis, S. Tapscott, M. Thayer, M. Krause, R. Benezra, T.K. Blackwell, D. Turner, R. Rupp, S. Hollenberg, Y. Zhuang, and A. Lassar. 1991. The myoD gene family: Nodal point during specification of the muscle cell lineage. Science 251: 761-766.

Wright, W.E. 1992. Muscle basic helix-loop-helix proteins and the regulation of myogenesis. Curr. Opin. Gen. Dev. 2: 243248.

Wright, W.E., D.A. Sassoon, and V.K. Lin. 1989. Myogenin, a factor regulating myogenesis, has a domain homologous to MyoD. Cell 56: 607-617.

Yee, S.-P. and P.W.J. Rigby. 1993. The regulation of myogenin gene expression during the embryonic development of the mouse. Genes \& Dev. 7: 1277-1289.

Yu, Y.-T., R.E. Breitbart, L.B. Smoot, Y. Lee, V. Mahdavi, and B. Nadal-Ginard. 1992. Human myocyte-specific enhancer factor 2 (MEF2) comprises a group of tissue restricted MADS box transcription factors. Genes \& Dev. 6: 1783-1798. 


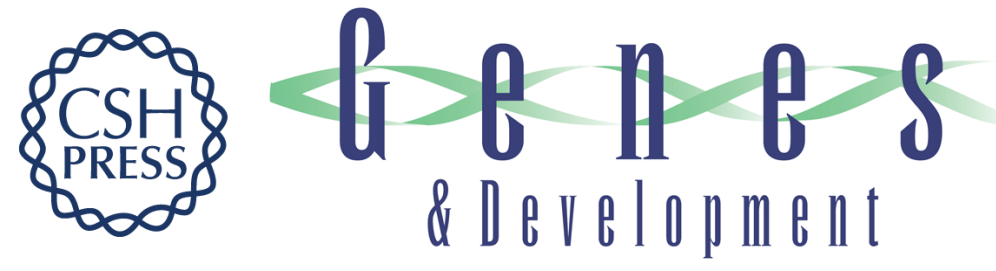

\section{bHLH factors in muscle development: dead lines and commitments, what to leave in and what to leave out.}

E N Olson and W H Klein

Genes Dev. 1994, 8:

Access the most recent version at doi:10.1101/gad.8.1.1

References This article cites 58 articles, 30 of which can be accessed free at:

http://genesdev.cshlp.org/content/8/1/1.full.html\#ref-list-1

License

Email Alerting

Service

Receive free email alerts when new articles cite this article - sign up in the box at the top right corner of the article or click here.

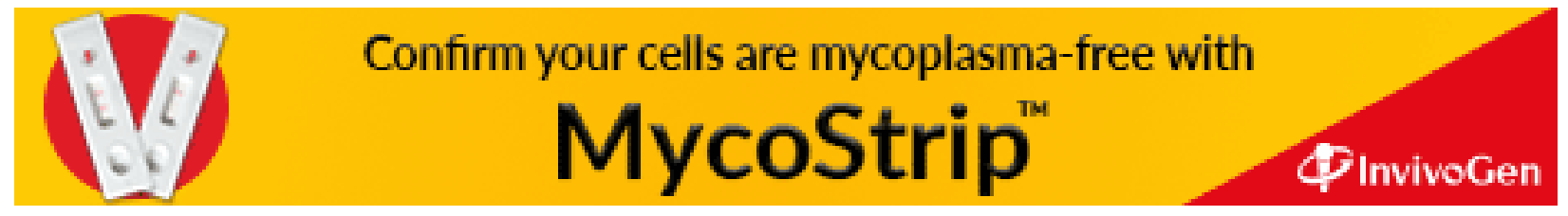

